\title{
PROGRAMAS NACIONAIS DE INICIAÇÃO À DOCÊNCIA NO BRASIL
}

\author{
Maria do Carmo de Sousa \\ mdcsousa@ufscar.br \\ https://orcid.org/0000-0002-5523-757X \\ Universidade Federal de São Carlos (UFSCar) \\ São Carlos, Brasil. \\ Renata Prenstteter Gama \\ rpgama@ufscar.br \\ https://orcid.org/0000-0001-6338-4345 \\ Universidade Federal de São Carlos (UFSCar) \\ São Carlos, Brasil.
}

Recibido: 01/11/2020 Aceptado: 01/12/2020

\begin{abstract}
Resumen
Este artículo es el resultado de una investigación cualitativa y se caracteriza como documental. Su objetivo es presentar y comprender, desde una perspectiva histórica, cómo los programas de iniciación a la docencia se han constituido en una política nacional de capacitación del profesorado, así como el rol que los programas han desempeñado en el contexto de la capacitación inicial del profesorado. El proceso de idealización e implementación de una política nacional para la iniciación a la docencia se constituye por dos programas públicos promovidos por la agencia nacional (Ministerio de Educación y Cultura - MEC) y vinculados a Instituciones de educación superior aprobadas por edicto público. Se completa doce años el primer Programa Institucional para Becas de Iniciación a la docencia (Pibid) y el segundo Programa de Residencia Pedagógica (PRP) se encuentra en su segundo año de implementación. En los resultados, pudimos identificar dos movimientos históricos principales en la constitución de la iniciación a la docencia en Brasil, el primero vinculado a la aproximación de la universidad y las escuelas y las contribuciones de Pibid a la política nacional de capacitación del profesorado y el segundo movimiento reflexivo sobre pasantías obligatorias y la implementación del PRP.

Palabras clave: Programa Institucional de becas de iniciación a la docencia (Pibid). Programa de residencia pedagógica (PRP). Capacitación inicial del profesorado. Política nacional para la capacitación del profesorado en Brasil. Capacitación del profesorado. Iniciación a la docencia.
\end{abstract}

\section{PROGRAMAS NACIONAIS DE INICIAÇÃO À DOCÊNCIA NO BRASIL}

\section{Resumo}

Esse artigo é decorrente de pesquisa qualitativa e caracterizada como documental. Tem como objetivo apresentar e compreender, a partir de uma perspectiva histórica, como os programas de iniciação à docência vêm se constituindo em uma política nacional de formação de professores, bem como, o papel que os programas têm desempenhado no contexto da formação inicial de professores. O processo de idealização e implementação de uma política nacional de iniciação à docência se constitui a partir de dois programas públicos fomentados pelo órgão nacional (Ministério de Educação e Cultura - MEC) e vinculados às instituições de ensino superior aprovadas em edital público. O primeiro Programa Institucional de Bolsas de Iniciação à docência (Pibid) completou doze anos e o segundo Programa Residência Pedagógica (PRP) está 
no seu segundo ano de implementação. Nos resultados pudemos identificar dois principais movimentos históricos da constituição da iniciação à docência no Brasil, sendo o primeiro vinculado a aproximação da universidade e escolas e as contribuições do Pibid para a política nacional de formação de professores e o segundo movimento reflexivo sobre os estágios obrigatórios e a implementação do PRP.

Palavras chave: Programa Institucional de Bolsa de Iniciação à Docência (Pibid). Programa de Residência Pedagógica (PRP). Formação Inicial de professores. Política Nacional de Formação de Professores no Brasil. Formação de professores. Iniciação à docência.

\title{
NATIONAL TEACHING INITIATION PROGRAMS IN BRAZIL
}

\begin{abstract}
This article is a result of qualitative research and characterized as documentary. It aims to present and understand, from a historical perspective, how the programs of initiation to teaching have been constituted in a national teacher training politic, as well as the role that the programs have played in the context of initial teacher formation. The process of idealization and implementation of a national politic of initiation to teaching is constituted from two public programs promoted by the national agency (Ministry of Education and Culture - MEC) and linked to institutions higher education approved in public notice. The first Institutional Initiation Scholarship Program teaching (Pibid) completed twelve years and the second Pedagogical Residency Program (PRP) is in its second year of implementation. In the results we were able to identify two main historical movements in the constitution of teaching initiation in Brazil, the first being linked to the approximation of the university and schools and the contributions of Pibid to the national politic of teacher formation and the second reflective movement on mandatory internships and the implementation of the PRP.

Keywords: Institutional Teaching Initiation Scholarship Program (Pibid). Pedagogical Residency Program (PRP). Initial teacher formation. Politics National Teacher Formation Program in Brazil. Teacher formation. Initiation to teaching.
\end{abstract}

\section{Introducción}

El problema de esta investigación se relaciona directamente con la capacitación del profesorado, especialmente con respecto a la configuración de las políticas nacionales de capacitación del profesorado que se han desarrollado en Brasil desde 2000 y comenzaron a implementarse, efectivamente, en 2007. En este contexto, se destacan dos programas nacionales de iniciación a la docencia: el Programa Institucional de Becas de Iniciación a la docencia (Pibid) que se implementó en 2008 y el Programa de Residencia Pedagógica (PRP) que comenzó a implementarse en 2018.

La necesidad de pensar en políticas nacionales de capacitación del profesorado ha sido señalada, durante muchos años, por investigadores brasileños, como Gatti y Freitas. Sus estudios muestran que la primera política nacional de capacitación del profesorado solo puede comenzar a aplicarse y implementarse cuando haya un contexto político favorable. Mientras se describía la política, Freitas (2007) hizo la siguiente advertencia: "La necesidad de una política global para capacitar y valorar a los profesionales de la educación que incluya, de manera articulada y prioritaria, capacitación inicial, capacitación continua y condiciones de trabajo, salarios y carreras, con la concepción socio-histórica del educador que la guía, hace que parte de las 
utopías e ideas de todos los educadores y las luchas por la educación pública en los últimos 30 años. Sin embargo, su realización no se materializa dentro de una sociedad marcada por la desigualdad y la exclusión que son características del capitalismo. Comprender estos lazos sociales es importante para no crear ilusiones de soluciones fáciles a los problemas de educación y capacitación" (Freitas, 2007, p. 1204).

Al ampliar la mirada a América Latina para comprender mejor el contexto brasileño que generó dicha política, en aquel momento, no se podía negar: "la baja calidad de la capacitación y la falta de condiciones adecuadas para ejercer el trabajo de los educadores se han desarrollado durante décadas, en nuestro país y en toda América Latina, de manera combinada, impactando en la calidad de la educación pública debido a la disminución de las inversiones públicas y condiciones de trabajo deterioradas para educadores y trabajadores de la educación (...)" (Freitas, 2007, p. 1204).

A pesar de las condiciones adversas y la mala calidad de la capacitación señalada, la misma autora hizo hincapié, en el momento en que estaba en "marcha una política de capacitación del profesorado (...) que corresponde al Estado ofrecer igualdad de oportunidades, opuesto a igualdad de condiciones, que se llevaría a cabo mediante el desarrollo de la capacitación exclusivamente en las universidades" (Freitas, 2007, p. 1206, 1207).

En el mismo razonamiento, los estudios de Gatti et al. (2011) llamaron la atención, al abordar las políticas de enseñanza a nivel federal brasileño, sobre la perspectiva de crear un sistema educativo nacional, ya que: "La configuración del gran aparato institucional establecido por el Ministerio de Educación (MEC), durante poco más de media década, describe una política nacional para la capacitación del profesorado guiada por la perspectiva de la institución de un sistema educativo nacional, que refleja el progreso del proceso de la responsabilidad de las autoridades públicas por el desempeño y la carrera de los docentes de educación básica y considera la capacitación como un proceso continuo para construir una práctica docente calificada y afirmar la identidad, el profesionalismo y la profesionalización de los docentes" (Gatti et al, 2011, p. 49).

De este modo, para que pudieran materializarse estas ideas, Gatti et al (2011) llamaron la atención sobre algunas acciones federales, o sea, institucionales, que culminaron en las siguientes creaciones: 1) Red nacional de educación continua para profesionales de Educación Básica; 2) programa de pro-alfabetización; 3) Programa de gestión del aprendizaje escolar (Gestar II); 4) Curso de Especialización en Educación Infantil; 5) Programa Proinfantil; 6) Programa de apoyo a planes de reestructuración y expansión de las Universidades federales (REUNI); 7) Universidad Abierta de Brasil (UAB) y Pro-licenciatura; 8) Programa Universidad para Todos (ProUni); 9) Plan nacional de capacitación del profesorado de la Educación Básica (PARFOR); 10) Programa Institucional para Becas de Iniciación a la docencia (Pibid), y las últimas cinco creaciones provienen de acciones políticas directamente relacionadas con las "políticas para la capacitación inicial del profesorado" (Gatti et al, 2011, p. 118).

Descubrimos, a partir de los estudios de Freitas (2007), Gatti et al (2011) y Locatelli (2018), que por primera vez en Brasil comenzó, de manera institucional bajo los dominios del gobierno federal, la configuración de una política para la capacitación del profesorado, que incluía la capacitación inicial del profesorado. Este hecho indicaba que no se olvidaría a los jóvenes que estaban interesados en cursos de licenciatura, de diferentes áreas del conocimiento. En consecuencia, se deben incluir las escuelas de educación básica.

Sin embargo, existe un problema por resolver: ¿cómo financiar dichas acciones y creaciones para que la implementación de la política nacional de capacitación del profesorado, entre ellas, la relacionada con la capacitación inicial del profesorado, pudiera llevarse a cabo considerando 
las particularidades y singularidades tanto de las universidades, y cuántas escuelas de educación básica estarían involucradas en todo el territorio brasileño?

Para responder a esta pregunta, las autoras antes mencionadas indicaron algunos elementos históricos que pudieron resolver el problema: la reconfiguración de la Coordinación para la Mejora del Personal de Educación Superior (Capes) y la publicación del Decreto número 21, en 2009.

Ha sido necesario redefinir la función de Capes, que hasta ese momento era una de las agencias brasileñas que promovía la investigación a nivel de posgrado. A partir de 2007, esta agencia de desarrollo, además de los estudios de posgrado, comenzó a regular la capacitación del profesorado y a actuar en Educación Básica, llegando a ser conocida como la "nueva" Capes con el propósito de: "inducir y fomentar, incluso en colaboración con los Estados, los municipios y el Distrito Federal, y exclusivamente a través de acuerdos con instituciones de educación superior públicas o privadas, la capacitación inicial y continua de profesionales de la enseñanza, respetando la libertad académica de las instituciones asociadas(...)” (Ley 11.512/07, art. 2, § 2)

Además de la reconfiguración de Capes, para establecer colaboraciones entre municipios, estados y el gobierno federal, así como entre las escuelas de Educación Básica y Universidades, se publicó en el Diario Oficial de la Unión (DOU), Sección 1, del 30 de enero de 2009, el Decreto de número 21, firmado por el entonces Presidente de la República, Luís Inácio Lula da Silva. El propósito del decreto era "organizar la capacitación inicial y continua de los profesionales de la enseñanza de la Educación Básica, en un régimen de colaboración entre la unión, los estados, el Distrito Federal y los municipios" (Brasil, 2009, p. 1). Se estableció que "los cursos para actualizar y especializar a los docentes estarían a cargo de Capes y deberían ser aprobados por su Consejo Técnico-Científico para la Educación Básica” (Brasil, 2009, p. 2).

En este contexto, la principal modalidad de enseñanza en la capacitación del profesorado sería presencial, aunque se reconoció la importancia de los sistemas semipresenciales y a distancia. Los aspectos más destacados serían: 1) "en el reconocimiento de que la capacitación del profesorado para todas las etapas de la Educación Básica es un compromiso público del Estado" y 2) "en la necesidad de articulación entre la educación inicial y continua, así como entre los diferentes niveles y modos de educación" (Brasil , 2009, p. 2).

Por lo tanto, una de las funciones de Capes, a partir de ese momento, ha sido fomentar la capacitación de profesionales de la enseñanza para trabajar en Educación Básica, incluido el fomento de programas de iniciación a la docencia y la concesión de becas a estudiantes matriculados en cursos de licenciatura de grado plena en instituciones de educación superior.

Por este motivo, de acuerdo con el Artículo 10, §10. del decreto, los programas de iniciación a la docencia deben prever: 1) "la articulación entre las instituciones de educación superior y los sistemas y redes de Educación Básica" y 2) "la colaboración de los estudiantes en las actividades de enseñanza-aprendizaje de la escuela pública" (Brasil, 2009, p. 2). Dichos programas, según el mismo artículo, §2o. del documento, solo pueden "contemplar cursos de licenciatura con evaluación positiva conducida por el MEC, bajo los términos de la Ley 10.861, del 14 de abril de 2004" (Brasil, 2009, p. 2).

Vale la pena también llamar la atención sobre el hecho de que, a pesar de todas las acciones, creaciones y regulaciones dirigidas a la profesionalización docente, indicadas en los párrafos anteriores, en el período de 2008 a 2018, solo Pibid se configuró en un programa nacional cuando alcanzó a la mayoría de las universidades públicas y privadas que tenían cursos de licenciatura, así como varias escuelas públicas de Educación Básica de todos los estados brasileños. En otras palabras, Pibid se implementó, desde el norte hasta el sur del país, a partir de 2008, a través de publicaciones de edictos. Diez años después, en 2018, durante el gobierno 
del presidente Michel Miguel Elias Temer Lulia, Capes creó un nuevo programa institucional, con miras a la iniciación a la docencia, el Programa de Residencia Pedagógica (PRP).

Así, en Brasil, tenemos la opción de crear dos programas destinados a la iniciación a la docencia y vinculados a la capacitación inicial del profesorado. Este escenario difiere de las políticas de apoyo para profesores principiantes descritas por Gatti et al (2011) en todo el mundo del Informe de la OCDE (2006) en el que identifica diez países que desarrollan programas nacionales de estímulo a la docencia con profesores con licencia en inicio de sus carreras profesionales (Australia, Corea del Sur, Francia, Grecia, Inglaterra, Gales, Irlanda del Norte, Japón, Suiza y Escocia).

A partir de los estudios que abordan el tema presentado hasta ahora, tanto de una política nacional de capacitación del profesorado como de los dos programas de iniciación a la docencia, en Brasil, delineamos dos temas que condujeron la investigación:

1) ¿Cómo se configuran los programas nacionales de iniciación a la docencia, especialmente Pibid y PRP en Brasil? y

2) ¿Qué rol cumplen estos dos programas en la capacitación inicial del profesorado de Educación Básica, ya que cubren las diferentes áreas del conocimiento?

Este artículo trata sobre la implementación y el papel de estos dos programas en la capacitación inicial del profesorado. En los siguientes ítems, pretendemos mostrar cómo se pueden responder las preguntas presentadas desde una perspectiva histórica.

\section{Base teórico-metodológica}

La investigación se llevó a cabo en el ámbito del proyecto de Pasantía, que tenía el título: "Estudio comparativo de políticas y prácticas institucionales para la iniciación e inserción a la docencia" e involucró a investigadores brasileños que trabajan en las siguientes universidades: Universidade Cidade de São Paulo (UNICID), Universidade Federal de Ouro Preto (UFOP), Universidade Federal de São Carlos (UFSCar) e Pontifícia Universidade Católica de São Paulo (PUC) y docentes del Centro Regional de Profesores (CeRP) del Litoral, de Uruguay. El objetivo general del proyecto ha sido llevar a cabo un estudio comparativo de políticas y prácticas institucionales para la iniciación e inserción a la docencia desde diferentes contextos de capacitación. Uno de los focos de la investigación abarca los programas nacionales de iniciación a la docencia en Brasil.

En este sentido, al iniciar la investigación, ha sido necesario analizar el contexto de origen de dichos programas, es decir, las políticas públicas para la capacitación del profesorado que comenzaron a entrar en vigencia en Brasil, en 2007. Al mismo tiempo, ha sido necesario investigar el concepto de iniciación a la docencia.

También se debe considerar que los fundamentos teóricos y metodológicos se apoyan en una perspectiva histórica y que, por esta razón, consideran los documentos consultados como fuentes históricas, especialmente aquellos que tienen como objeto de investigación las políticas nacionales para la capacitación del profesorado y iniciación a la docencia. Según D'Ambrósio (1999): "Una vez que se han identificado los objetos del estudio, la lista de hechos, fechas y nombres depende de los registros, que pueden resultar de una naturaleza muy diferente: memorias, prácticas, monumentos y artefactos, escritos y documentos. Estas se llaman fuentes históricas y la interpretación depende de la ideología, en forma de una filosofía de la historia (...)“(D’Ambrósio, 1999, p. 4).

Así, tenemos el objetivo de presentar y comprender, desde una perspectiva histórica, cómo los programas de iniciación a la docencia se han constituido en una política nacional de 
capacitación del profesorado, así como el rol que los programas han desempeñado en el contexto de la capacitación inicial del profesorado que comenzó a perfilarse desde principios de la década de 2000.

\section{Metodología de investigación desarrollada}

Esta investigación cualitativa y teórica puede caracterizarse como documental, inspirada en "estudios típicamente históricos", como señalan Fiorentini y Lorenzato (2007, p. 103), considerando que, para recopilar la información necesaria, utilizamos fuentes primarias, tales como: textos teóricos, investigaciones, registros y libros que tratan de temas que involucran políticas públicas para la capacitación del profesorado y programas de iniciación a la docencia.

Al desarrollarla, elegimos una metodología que se compuso por los siguientes momentos y estrategias:

$1^{\circ}$.) La realización de análisis histórico del contenido. Consistió en un estudio teórico sobre la historia de los conceptos tratados en las políticas nacionales de capacitación del profesorado, con atención particular a dos programas de iniciación a la docencia. El estudio necesariamente se refería a una investigación bibliográfica que involucraba tanto investigaciones sobre la capacitación del profesorado como las relaciones entre la capacitación del profesorado y los programas de iniciación a la docencia.

$2^{\circ}$.) Estudio teórico del concepto de iniciación a la docencia en el contexto brasileño. Consistió en un estudio sobre el concepto de iniciación a la docencia defendido por Silveira (2015 y 2017).

Los momentos y estrategias que compusieron la metodología, nos hacen estar de acuerdo con los estudios de Lanner de Moura (1995) cuando indican que, para lograr resultados que promuevan el avance del área de conocimiento en la que se inserta el problema, es necesario tener una estrecha articulación entre contenido de investigación y metodología. Por lo tanto, concebimos que, si la teoría se está construyendo en el proceso de investigación, debería ocurrir un movimiento idéntico con la metodología.

En este sentido, el método no es algo externo a la investigación, es ajustable a él como un traje se ajusta al cuerpo, pero consiste en ideas y acciones que tejen coherentemente todos los elementos de la investigación. Es lo que garantiza la no separación entre el conocedor (el investigador), el conocimiento (que se construirá a través de la investigación) y lo conocido (el conocimiento ya producido sobre el tema de investigación) de una manera que combine todos estos elementos en un conocimiento no fragmentado de la realidad investigada.

Así, destacamos también la necesidad del tercer momento y la estrategia:

$3^{\circ}$.) Consulta al portal de la revista de Capes para identificar los artículos científicos publicados sobre los programas de iniciación a la docencia en Brasil.

En esta búsqueda, basada en dos descriptores: 1) Iniciación a la docencia y 2) Política pública, encontramos artículos científicos brasileños revisados por pares (68) entre los años 2008 a 2019. En el segundo paso, buscamos los artículos completos en las revistas indicadas en la búsqueda y obtuvimos acceso a 50 artículos. Después de la primera lectura, separamos los artículos por el foco principal: 
Cuadro 1: Artículos científicos brasileños - iniciación a la docencia y políticas públicas

\begin{tabular}{|l|c|}
\hline \multicolumn{1}{|c|}{ ENFOQUE DE ESTUDIO } & CANTIDAD \\
\hline Programa Institucional para Becas de Iniciación a la docencia & 16 \\
\hline Política y gestión educativa & 5 \\
\hline $\begin{array}{l}\text { Educación Superior, Educación Profesional, Instituto Federal de } \\
\text { Educación }\end{array}$ & 2 \\
\hline Inclusión social & 6 \\
\hline Educación y salud & 8 \\
\hline Aspectos didácticos & $\mathbf{5 0}$ \\
\hline TOTAL & 2 \\
\hline
\end{tabular}

Fuente: Preparado por los autores.

A partir de esta identificación, pudimos seleccionar los artículos que trataban explícitamente en sus datos sobre el proceso de iniciación a la docencia y/o la política pública involucrada (19).

En una segunda búsqueda, usando los descriptores: 1) Programa Residencia pedagógica en capacitación del profesorado y 2) Iniciación a la docencia. Encontramos 13 artículos científicos relacionados con PRP. Entre ellos tuvimos cinco artículos relacionados con el enfoque de este artículo, dos de los cuales presentan la historia del programa en Brasil y tres que discuten el proceso de iniciación a la docencia.

El análisis de datos siguió una línea interpretativa cuya característica es: "particularización, en lugar de generalización de resultados. La búsqueda no es para universales abstractos, que se alcanza, según Moreira (1990), a generalizaciones estadísticas, pero más bien a universales concretos, que se logra a través del estudio detallado de un caso específico, ubicado culturalmente" (Lanner de Moura, 1995, p. 69).

Por esta razón, la interpretación nos ha permitido organizar ejes temáticos que tienen como objetivo principal mostrar cómo los programas de iniciación a la docencia se han implementado en Brasil y el rol que han desempeñado en la capacitación inicial del profesorado de Educación Básica.

\section{Breve historia sobre la implementación y el rol de Pibid como política pública de capacitación del profesorado}

El primer edicto público de Pibid, publicado el 12 de diciembre de 2007 y firmado por el Ministro de Educación, Fernando Haddad. Al definir cuál sería el programa, se hizo hincapié en la acción conjunta que debería llevarse a cabo entre el Ministerio de Educación, a través de la Secretaría de Educación Superior - SESu, la Fundación de Coordinación para la Mejora del Personal de Educación Superior - CAPES y el Fondo Nacional de Desarrollo de la Educación FNDE, "con el fin de fomentar la iniciación a la docencia de estudiantes de instituciones federales de educación superior y preparar la capacitación del profesorado a nivel superior" (Brasil, 2007, p. 1). Este aviso se destinaba a las áreas de Química, Física, Biología y Matemáticas y, según el director nacional "las áreas iniciales elegidas se justificaron debido a la baja demanda de estos títulos, así como a la alta tasa de deserción en estos cursos. Además, 
el número de profesores en estas áreas no satisfizo la demanda de las escuelas que, a su vez, ofrecían a profesionales en otros campos los roles de docentes en el campo de las ciencias y las matemáticas" (Silveira, 2017, p.52).

Según Silveira (2017), todavía no había una definición segura de lo que podría significar el concepto de iniciación a la docencia. Se puede decir que el rol principal del programa sería el establecimiento de colaboraciones entre universidades y escuelas de Educación Básica, así como entre Capes, estados y municipios y la valorización del magisterio.

Durante los años de 2009, 2010, 2011, 2012, 2013, 2018 y 2020, se publicaron edictos (Brasil, 2020b) en continuidad con el Programa. En estos edictos, hubo una expansión de áreas e instituciones autorizadas para enviar propuestas, como en todas las IES que tuvieron cursos de licenciatura como en 2009 IES federales y estatales; IES federales, estatales y comunitarias de 2010 y 2011 y 2014. Pibid se ha expandido rápidamente, de casi tres mil becarios de licenciatura en 2008 a noventa mil en 2013 distribuidos en todo Brasil. Según Gatti (2011), el número de instituciones que presentaron sus propuestas creció, según los avisos de Capes, lo que revela, al menos, entusiasmo por esta política, dado que los requisitos son relativamente fuertes. (Gatti et al, 2011).

Se observa que, en los años de 2014 a 2017, no se publicó ningún edicto público. En ese período, el programa tenía riesgo de desaparecer, aunque se ha evaluado positivamente después de sus primeros años de implementación. En 2014, el propio CAPES realizó un estudio evaluativo en colaboración con investigadores en el área de Educación, basado en cuestionarios enviados a los participantes del Programa que señalaron que: "Los testimonios son muy positivos, en la gran mayoría; los detalles ofrecidos para justificar esta positividad son numerosos y se expresan en las contribuciones descritas. Las mejoras deben implementarse en vista del valor atribuido a su metodología y a las afirmaciones insistentes sobre la necesidad imperiosa de su continuación debido a su rol de otorgar valor a las licenciaturas en las IES, de calificarlas mejor y a sus profesores, de proporcionar una mejor capacitación para los futuros profesores de la educación básica, para aportar contribuciones a los profesores supervisores y sus escuelas, a la enseñanza a través de la creatividad didáctica. Sin duda, debido a los datos analizados hasta ahora, este es un programa altamente efectivo con respecto a la capacitación inicial del profesorado" (Gatti et al, 2014, p.103).

Se coordenó otro estudio realizado en el momento de la incertidumbre sobre la continuidad de Pibid (2016) por André (2018), en continuidad con el estudio evaluativo en colaboración con CAPES. La investigación desarrollada se caracterizó por el tipo survey y tuvo como objetivo analizar el proceso de inserción profesional de profesores principiantes, graduados de tres programas de iniciación a la docencia: Programa institucional para becas de iniciación a la docencia, beca de alfabetización y residencia pedagógica en la Universidade Federal de São Paulo/Guarulhos. Según ese estudio: "Hubo una acusación, del Ministro de Educación, de que pocos graduados de Pibid estaban trabajando en educación, de ahí que el retorno esperado para las redes educativas no compensara los recursos financieros gastos. Una declaración pública del ministro, Aloizio Mercadante, afirmó que menos del 20\% de los ex-pibidianos se encontraban en la escuela. En ese momento, era esencial contar con los datos de la encuesta que indicaban que, en 2016, el $67 \%$ de los graduados trabajaban como docentes en educación básica, una gran parte de ellos en las escuelas públicas (61\%), lo que reveló una situación muy diferente a la mencionada por el ministro. Se enviaron los resultados de la encuesta inmediatamente por el coordinador de investigación a los gerentes responsables de administrar Pibid en el Ministerio de Educación (MEC) y corroboraron las afirmaciones del grupo de intelectuales (Fórum dos 
Coordenadores Institucionais do Pibid - FORPIBID) que lucharon por la permanencia del programa" (André, 2018, p. 17).

Hay otros documentos que también fueron relevantes y publicados en este momento de incertidumbre. Debido a las proporciones que alcanzó el programa, se implementó la Ley $\mathrm{N}^{\circ}$ 12.796/2013 (Brasil, 2013) de Directrices y Bases Educativas, que a partir de mayo de 2013 incorporó el programa como una política de capacitación del profesorado y la Ordenanza $\mathrm{N}^{\circ}$ 096, del 18 de julio de 2013 de Capes que presentó, por primera vez, el principio de iniciación a la docencia, en el Art. 4: "ayudar a los estudiantes de licenciatura a insertarse en la cultura docente del magisterio, a través de la apropiación y reflexión sobre instrumentos, conocimientos y peculiaridades del trabajo docente".

Según Silveira, "la inducción de Capes es que el alcance de los proyectos considera diferentes características y dimensiones de la iniciación a la docencia. En otras palabras, diferentes características y dimensiones de una cultura: la docencia" (Silveira, 2015, p. 364). Por lo tanto, se entendió que la iniciación a la docencia sería una forma de apropiarse de diferentes elementos que constituyen la cultura de la docencia, haciendo que los estudiantes tengan la oportunidad de conocer matrices teóricas, desarrollar reflexiones sobre la docencia, producir instrumentos didáctico-pedagógicos y apropiarse de una forma de hacer, pensar y actuar en las escuelas de educación básica (Silveira, 2017), y el concepto de iniciación a la docencia se relacionó con: "La importancia de que el alumno participe en diferentes acciones cuya intencionalidad pedagógica favorecería la reconstrucción de conceptos sobre la práctica profesional y las prácticas didácticas" (Silveira, 2017, p. 59).

Teniendo en cuenta que la definición de iniciación a la docencia hecha por Silveira $(2015 ; 2017)$ se centra en la cultura de la profesión docente, analizamos los edictos publicados en 2018 y 2020, durante el gobierno del presidente Jair Messias Bolsonaro. Podríamos verificar cambios estructurales en la composición de los proyectos, tales como: requisito de vincular el plan de actividades y/o estrategias de articulación con el conocimiento de la Base Nacional Común Curricular (BNCC) y el público objetivo, dirigiéndose a los graduados de la primera mitad del curso. También hubo un cierto cambio en relación con la definición del programa que estaba presente en todos los avisos publicados hasta esa fecha. En este nuevo contexto: "Pibid es un programa de la Política Nacional de Capacitación del Profesorado del Ministerio de Educación (MEC) que tiene como objetivo proporcionar a los estudiantes de cursos de licenciatura su inserción en las escuelas públicas de educación básica. Para el desarrollo de proyectos institucionales para la iniciación a la docencia, el programa otorga becas a estudiantes de licenciatura, profesores de escuelas públicas en educación básica y profesores de IES" (Brasil, 2020b, p. 1).

En otras palabras, si antes el concepto de iniciación a la docencia estaba vinculado a una cultura específica de la profesión docente, Pibid comienza a considerar la vida cotidiana de las escuelas en estos dos últimos edictos.

Al analizar las inscripciones de las universidades para participar en el último edicto, Capes publicó la siguiente declaración en la página del programa: "294 instituciones de educación superior (IES) completaron la finalización del proyecto institucional para la iniciación a la docencia en Sicapes, especialmente al Edicto No. 02/2020 - Pibid" (Brasil, 2020b, p. 1). De estos, solo 250 instituciones tendrán sus proyectos contemplados. En otras palabras, en comparación con 2011, la adhesión al programa continuó aumentando.

Al analizar los artículos científicos identificados, se puede decir que durante todos estos diez años de Pibid, hay una gran expectativa por parte de las instituciones brasileñas de que se valoren los títulos universitarios y que el Programa contribuya desde la aproximación con el 
campo profesional desde el comienzo del curso, permitiendo la maduración de las opciones de los graduados.

También podemos indicar que no es por nada que, cuando el Programa está en riesgo de desaparecer, las universidades se mueven para salvarlo y mantenerlo como una política estatal que permite a los estudiantes de grado sumergirse en las escuelas públicas y saber, reflexionar, cuestionar y apropiarse de los elementos que conforman la profesión docente.

\section{Breve historia de la implementación y el rol de PRP como política pública para la capacitación del profesorado}

A diferencia de Pibid, el PRP enfrentó diferentes desafíos cuando se instituyó, como una política pública, en el contexto de la capacitación del profesorado. Los artículos científicos identificados en el mapeo indicaron las tensiones político-pedagógicas que ocurrieron en ese período y destacaron tanto la hegemonía del Estado como los "avances prácticos en el conocimiento y las habilidades específicas de valorización profesional y una postura de las IES" (Barbosa y Dutra, 2019 , p. 156) y la resistencia de los educadores al programa.

Al describir el historial de implementación, Silva y Cruz (2018) afirman que: "El tema de la residencia en el área de Educación no es una discusión nueva en Brasil y también ha surgido bajo diferentes nomenclaturas (...). Era una concepción de una modalidad posterior a la capacitación inicial que ha sido llamada de Residencia Educativa, que incluía para este propósito una propuesta para enmendar el artículo 65 de LDBEN/96 (...)" (Silva y Cruz, 2018, p. 230).

Como el proyecto no se aprobó en el Senado, cinco años después hubo un nuevo intento de implementar el concepto de Residencia, en el área de Educación. En un primer momento, el concepto de residencia estaba directamente relacionado con la educación continua, después, se dio cierta importancia a los futuros maestros, es decir, capacitación inicial: "En 2012, un proyecto del senador Blairo Maggi (PR-MT) adapta el PLS 227 propuesto por Marco Maciel (DEM-PE) en 2007. La reformulación de Maggi, Proyecto de ley (PLS) $n^{\circ}$ 284/12, trajo el nombre de Residencia Pedagógica, que sería "una etapa más de la capacitación inicial para la enseñanza en la educación infantil y en los primeros años de la escuela primaria, en forma de 'residencia', pagado a través de becas y con una carga de trabajo mínima de 800 horas. (...) existiría la posibilidad de que el certificado de aprobación en la Residencia Pedagógica pudiera utilizarse en los procesos de selección de las redes educativas, en el contexto de concursos para exámenes y presentación de títulos, así como, los profesores en ejercicio, pudieran beneficiarse de la residencia, como estrategia de actualización profesional. Más tarde, en 2014, la Comisión de Educación, Cultura y Deporte del Senado aprobó el proyecto de ley 6/2014, PLS 6/2014 del senador Ricardo Ferraço (PSDB-ES), que propuso la enmienda de la LDB, proponiendo la Residencia Docente (...) La propuesta preveía que los sistemas educativos ofrecerían la residencia docente a graduados en un número igual o superior al $4 \%$ del personal docente respectivo en actividad hasta el año 2024, y deberían garantizar hasta 2017 el número de vacantes correspondientes al mínimo del 0,5\%"(Silva y Cruz, 2018, p. 230, 231).

Los mismos autores también indican que, "aunque los proyectos de ley propuestos en el Senado no se han implementado, experiencias de residencia aisladas en el campo de la formación docente en los sistemas municipales, estatales y federales de educación básica y superior han estado ocurriendo desde la década de 2000" (Silva y Cruz, 2018, p. 232). Estas iniciativas se pueden dividir en dos ejes: "Residencia en capacitación inicial relacionada con pasantías comúnmente llamadas de residencia pedagógica" y "residencia como educación 
continua comúnmente conocida como residencia docente", como se muestra en la tabla a continuación:

Cuadro 2: Ejes relacionados con el concepto de residencia

Eje 1: Residencia en capacitación inicial relacionada con pasantías comúnmente llamada de residencia pedagógica

\begin{tabular}{|c|c|c|c|c|}
\hline 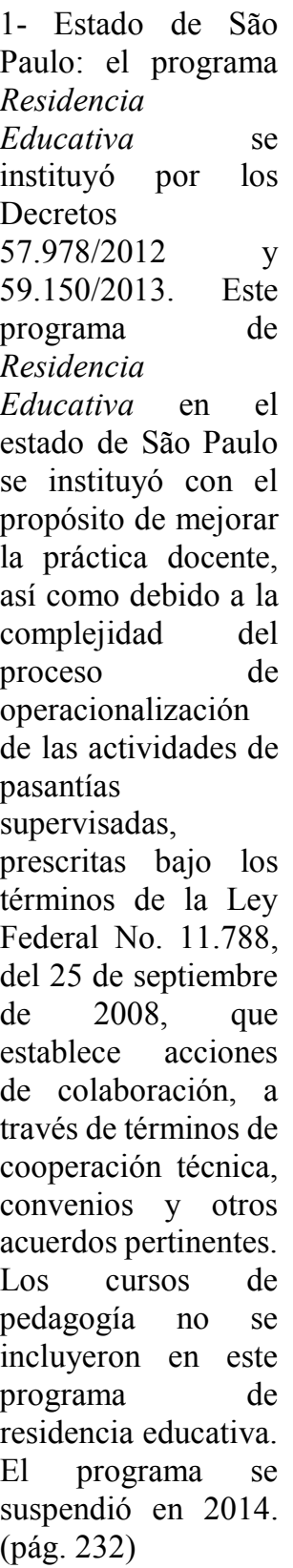 & $\begin{array}{l}\text { 2- La Escola } \\
\text { de Filosofia, } \\
\text { Letras e } \\
\text { Ciências } \\
\text { Humanas da } \\
\text { Universidade } \\
\text { Federal de } \\
\text { São Paulo } \\
\text { (EFLCH/Unif } \\
\text { esp-Campus } \\
\text { Guarulhos) ha } \\
\text { desarrollado } \\
\text { desde 2009, } \\
\text { en el curso de } \\
\text { Pedagogía, la } \\
\text { Residencia } \\
\text { Pedagógica } \\
\text { en la } \\
\text { perspectiva de } \\
\text { la pedagogía } \\
\text { de } \\
\text { alternancia, } \\
\text { que consiste } \\
\text { en un } \\
\text { programa de } \\
\text { pasantía. } \\
\text { Caracterizado } \\
\text { según el } \\
\text { proyecto, por } \\
\text { un contacto } \\
\text { sistemático } \\
\text { progresivo } \\
\text { entre } \\
\text { estudiantes, } \\
\text { docentes de la } \\
\text { IES y las } \\
\text { escuelas de } \\
\text { educación } \\
\text { básica. (pág. } \\
232-233 \text { ) }\end{array}$ & $\begin{array}{l}\text { 3- En Jundiaí (SP), un } \\
\text { proyecto similar a la } \\
\text { residencia pedagógica } \\
\text { entró en vigencia en } \\
\text { 2014, pero no hubo } \\
\text { continuidad. } \\
\text { iniciativa provino del } \\
\text { ayuntamiento local al } \\
\text { asociarse con tres } \\
\text { universidades privadas } \\
\text { de la ciudad. Este es el } \\
\text { Programa de pasantías } \\
\text { remuneradas, que } \\
\text { ofrece salarios por un } \\
\text { monto de R\$ 950.00 } \\
\text { para 225 pasantes en } \\
\text { las áreas de pedagogía, } \\
\text { letras, psicología y } \\
\text { educación física. Con } \\
\text { una carga de trabajo de } \\
\text { cinco horas al día, los } \\
\text { pasantes no solo } \\
\text { ayudan a los } \\
\text { educadores de la red en } \\
\text { sus actividades } \\
\text { escolares diarias, como } \\
\text { la planificación del } \\
\text { contenido, sino que } \\
\text { también experimentan } \\
\text { la rutina de las } \\
\text { unidades escolares. } \\
\text { Además de participar } \\
\text { en el aula, también } \\
\text { aprenden conceptos } \\
\text { relacionados con la } \\
\text { gestión (p. 233). }\end{array}$ & $\begin{array}{l}\text { 4- En el } \\
\text { Instituto de } \\
\text { Educación } \\
\text { Superior } \\
\text { Ivoti (ISEI), } \\
\text { ubicado en el } \\
\text { municipio de } \\
\text { Ivoti, en Rio } \\
\text { Grande do } \\
\text { Sul (RS), el } \\
\text { Proyecto de } \\
\text { Residencia } \\
\text { Pedagógica } \\
\text { creado en } \\
\text { 2008 y aún } \\
\text { vigente, } \\
\text { prevé la } \\
\text { migración de } \\
\text { jóvenes } \\
\text { inscritos en la } \\
\text { institución, a } \\
\text { otras } \\
\text { ciudades del } \\
\text { país. } \\
\text { Realizan una } \\
\text { pasantía } \\
\text { supervisada } \\
\text { de una } \\
\text { semana en las } \\
\text { escuelas de la } \\
\text { red Sinodal } \\
\text { de Educación } \\
3 \text { y, durante } \\
\text { este período, } \\
\text { permanecen } \\
\text { en los } \\
\text { hogares de } \\
\text { los docentes } \\
\text { en cada } \\
\text { unidad (pág. } \\
\text { 233). }\end{array}$ & 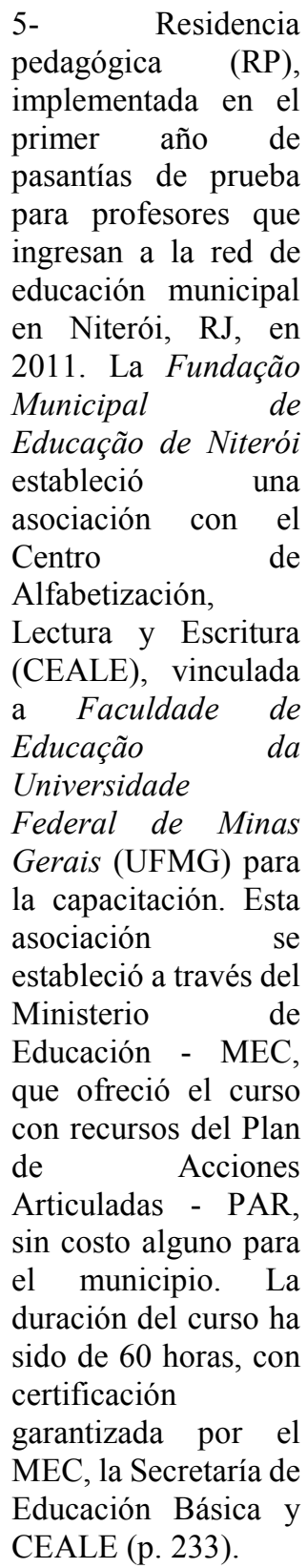 \\
\hline
\end{tabular}

Eje 2: Residencia como capacitación continua comúnmente llamada de residencia docente 
1- Capes- Dirección de educación básica- Proyectos especiales para apoyar la educación básica- escuelas de referencia- certificado de especialización: Colégio Pedro II (Río de Janeiro) - y Centro Pedagógico da Universidade Federal de Minas Gerais (Belo Horizonte) - La propuesta es equivalente al curso de posgrado lato-sensu y tiene como objetivo capacitar al profesor recién graduado, desarrollando estrategias pedagógicas asociadas con el trabajo académico (investigación), buscando mejorar la capacidad profesional adquirida en la graduación, mejorando el desempeño de este profesor en actividades prácticas y en todo el entorno de la escuela pública ( p. 235).
2- Colégio Visconde de Porto Seguro 5, ubicado en Morumbi, São Paulo - SP. El "Programa de Residencia Docente" es un proyecto piloto que comenzó en 2015. La escuela lo desarrolla tomando inspiración del Referendariat, un curso de capacitación del profesorado que se desarrolla en Alemania. Tiene una duración de un año y está dirigido a profesores y futuros profesores que tengan la intención de trabajar en el Colegio Visconde de Porto Seguro, en la escuela primaria y secundaria (p. 235).

\section{Fuente: (Silva y Cruz, 2018, p. 233-235).}

Así, durante algunos años, más precisamente, en el período de 2014 a 2018, la definición del concepto de residencia en la capacitación del profesorado de Educación Básica, pasó por varios momentos y consideró varias ideas, que solo podían presentarse cuando Faria y Diniz-Pereira (2019) se hizo la siguiente pregunta: “Residencia pedagógica: después de todo, ¿qué es?” y realizó una encuesta de investigación, proyectos de ley y experiencias que involucraron algún tipo de capacitación práctica de profesores que, en Brasil, se inspiró en alguna idea de residencia pedagógica. Después de analizar los documentos y, en consecuencia, concluir la investigación, Faria y Diniz-Pereira (2019) concluyen que: "La última propuesta de residencia relacionada con la capacitación inicial, anterior al Programa de Residencia Pedagógica MEC actual, se encontró en dos trabajos (Nogueira et al., 2011, 2013) (...)". Para los autores, el término residencia pedagógica se utilizó en el proyecto de investigación "siguiendo el modelo de programa para la capacitación de médicos, pero dirigido al universo de la pedagogía, lo que significa una implicación real con la rutina escolar, el aula y el entorno que componen la complejidad de la capacitación del profesorado". (Nogueira et al., 2011, p. 38) (Faria y Diniz-Pereira, 2019, p. 343).

Pensemos que el primer curso de licenciatura a introducir alguna idea de residencia pedagógica en la capacitación inicial ha sido la Pedagogía. Hasta entonces, los otros grados ni siquiera han sido mencionados. Lo mismo ocurre con la definición de lo que podría ser, el concepto de iniciación a la docencia, en esta nueva forma de pensar el curso de Pedagogía.

Por lo tanto, en base al análisis de propuestas puntuales y específicas, presentadas tanto en la Tabla 2 como en los estudios de Faria y Diniz-Pereira (2019), encontramos que seis se centraron en la iniciación a la docencia. Sin embargo, a pesar de esto, Capes lanzó el 18/10/2017, sin considerar necesariamente las experiencias acumuladas en todos estos años por las universidades y escuelas de Educación Básica que estuvieron involucradas en estas propuestas, un programa nacional llamado de Programa de Residencia Pedagógica (PRP), para todas las licenciaturas, durante el gobierno del Presidente Michel Miguel Elias Temer Lulia, bajo la dirección del Ministro de Educación, José Mendonça Bezerra Filho.

Una de las primeras menciones que se hicieron al PRP, en la página del MEC, indicó que el programa representaba una cierta modernización en relación con Pibid.

"La residencia pedagógica es parte de la modernización del Programa Institucional para Becas de Iniciación a la docencia (Pibid) y trae novedades, como la capacitación del estudiante de graduación, que tendrá una pasantía supervisada a partir del tercer año del título en durante todo el curso, en la escuela de educación básica. El objetivo principal es mejorar la calidad de 
la capacitación inicial y una mejor evaluación de los futuros docentes, que tendrán un seguimiento periódico. Se requiere que el programa se asocie con instituciones de capacitación y acuerdos con redes de educación pública" (Brasil, 2017, página de inicio).

Por primera vez, el concepto de residencia pedagógica en la capacitación inicial comienza a insertar el término pasantía supervisada. Este es el elemento principal que diferencia a Pibid de PRP. Por lo tanto, el nuevo programa, a diferencia de Pibid, que comenzó a considerar las Prácticas como componentes curriculares (PCC), el PRP ha sido diseñado para vincularse directamente a las pasantías obligatorias, de todos los cursos de licenciatura, como se indica en el primer edicto Capes no. 06/2018, publicado el 01/03/2018, cinco meses después de su lanzamiento: "La residencia pedagógica es una actividad de capacitación llevada a cabo por un estudiante regularmente matriculado en un curso de licenciatura y desarrollado en una escuela pública de educación básica, llamada escuela-campo" (Brasil, 2018, p. 1, 2).

A partir de la definición del concepto de residencia pedagógica, se establecieron los siguientes objetivos para el programa: "I. Mejorar la capacitación de los estudiantes en cursos de licenciatura, a través del desarrollo de proyectos que fortalezcan el campo de la práctica y lleven al estudiante a ejercer activamente la relación entre la teoría y la práctica docente profesional, utilizando la recopilación de datos y el diagnóstico sobre la enseñanza y aprendizaje escolar, entre otras didácticas y metodologías; II. Inducir la reformulación de la pasantía supervisada en cursos de licenciatura, basada en la experiencia de la residencia pedagógica; III. Fortalecer, expandir y consolidar la relación entre la IES y la escuela, promoviendo la sinergia entre la entidad que se forma y la que recibe el egreso de la licenciatura y estimulando el protagonismo de las redes de enseñanza en la capacitación del profesorado. IV. Promover la adaptación de los planes de estudio y las propuestas pedagógicas de los cursos de capacitación inicial del profesorado de educación básica a los lineamientos de la Base Nacional Común Curricular (BNCC)" (Brasil, 2018, p. 1)

Se preparó el Anexo III del edicto, con el fin de presentar las "referencias para la preparación del proyecto institucional para la residencia pedagógica" (Brasil, 2018, p. 17) por parte de las universidades calificadas para integrarse en el programa. El ítem tres, titulado "la Residencia Pedagógica", presentó directrices que indujeron cambios en pasantías obligatorias en cursos de licenciatura.

Aquí, el concepto de iniciación a la docencia no se ha definido y el papel del programa ahora se confunde con la pasantía supervisada. Dada la definición de Capes, residencia pedagógica, sinónimo de pasantía obligatoria, la resistencia al nuevo programa ha sido notable. Dejaron en claro en ese momento que las universidades deberían inscribirse en el programa.

En este primer edicto, había una indicación de que se contemplarían 350 instituciones. Se inscribieron 245. Quedan 105 lugares. Al final del proceso de selección, del total de 245 instituciones registradas, 242 se calificaron (Brasil, 2018). Este hecho no se repitió en 2020, como lo mostraremos en los siguientes párrafos. Aparentemente, la resistencia al PRP comenzó a caer, por varias razones, entre ellas, la falta de fondos para becas para estudiantes de licenciatura, especialmente para cursos de licenciatura.

No es de extrañar que el número de universidades interesadas en el segundo edicto de Capes no. 01/2020, publicado el 6 de enero de 2020, durante el gobierno del presidente Jair Messias Bolsonaro, ha sido mayor que en 2018. Este hecho puede confirmarse en la publicación realizada por Capes el 20/03/2020: han sido "270 Instituciones de Educación Superior (IES) las que completaron la finalización del proyecto institucional para la residencia pedagógica en Sicapes, de acuerdo con el Edicto No. 01/2020 - Residencia pedagógica” (Brasil, 2020a, p. 1). Es de destacar que el edicto indica que solo 250 instituciones tendrán contemplados sus proyectos. En 
otras palabras, en relación con 2018, hubo un aumento significativo, en las instituciones, por el programa.

Al leer el edicto, pudimos ver que hubo un cierto cambio en relación con la definición del programa, en comparación con el anuncio de 2018. Hay una cierta similitud entre lo que debería ser PRP y Pibid en 2020: "El Programa de Residencia Pedagógica es una de las acciones que forman parte de la Política Nacional de Capacitación del profesorado y tiene como objetivo inducir la mejora de la capacitación práctica en los cursos de licenciatura, promoviendo la inmersión del alumno en la escuela de educación básica, a partir del segundo semestre de su curso" (Brasil, 2018, página de inicio).

Además del objetivo anterior, se definieron otros cuatro: I - alentar la capacitación del profesorado en un nivel superior para la educación básica, lo que lleva al graduado a ejercer activamente la relación entre la teoría y la práctica docente profesional; II - promover la adaptación de los planes de estudio y las propuestas pedagógicas de los cursos de licenciatura a las directrices de la Base Nacional Común Curricular (BNCC); III - fortalecer y ampliar la relación entre las Instituciones de Educación Superior (IES) y las escuelas públicas de educación básica para la capacitación inicial del profesorado de educación básica; y IV - fortalecer el papel de las redes educativas en la capacitación de futuros docentes" (Brasil, 2018, página de inicio).

Aquí, el concepto de residencia pedagógica se separa una vez más de la pasantía obligatoria, de los cursos de licenciatura y una de las funciones del programa, es fortalecer las redes de enseñanza en el contexto de la capacitación inicial del profesorado, siempre que las colaboraciones entre las escuelas de educación básica y las universidades pueden establecerse institucionalmente.

\section{Consideraciones finales}

Al analizar cómo los programas nacionales brasileños, Pibid y PRP, de iniciación a la docencia se configuran e implementan en Brasil, como una política pública, encontramos que se está tejiendo una red de relaciones, ya que la capacitación docente en Brasil se realiza en las universidades.

Dicha red implica, por ejemplo, la disponibilidad política y la capacidad de inversión en el área de Educación, especialmente con respecto a la capacitación del profesorado, así como el establecimiento de colaboraciones institucionales entre las escuelas y universidades de Educación Básica; municipios, estados, gobierno federal y Capes.

En este movimiento, el de establecer colaboraciones institucionales, hay idas y venidas, avances y contratiempos que se materializan con cada aviso público emitido por Capes. No hay forma de negar la hegemonía del estado, especialmente cuando parece que, a partir de 2018, cuando hay cambios significativos en el contexto político brasileño, hay una insistencia en vincular, por ejemplo, los dos programas a la Base Nacional Curricular Común (BNCC) en los proyectos propuestos. El gobierno federal cuestiona la autonomía de las universidades y las escuelas de educación básica.

En el caso de Pibid, la historia de la implementación del programa muestra que, a pesar de esto, existe un consenso entre los edictos cuando se define que el programa no es una pasantía $\mathrm{y}$, por esta razón, debe permitir la inserción de todos los estudiantes de cursos de licenciatura en el programa, desde el comienzo del curso. Al mismo tiempo, durante todos estos años, el concepto de iniciación a la docencia ha madurado, teóricamente, en la medida en que se han completado las investigaciones sobre el programa y Capes ha valorado las contribuciones de las instituciones involucradas. El papel de Pibid es insertar a los futuros docentes en la cultura de 
la capacitación del profesorado, a través de actividades y acciones vinculadas a la práctica como un componente curricular (PCC).

La historia de la implementación de PRP, por otro lado, muestra algunas divergencias que ocurren en la definición del concepto en sí, ya que, al principio, el concepto de residencia se relacionaba con el área de la medicina. La pregunta que se ha hecho durante años ha sido: ¿cómo se puede insertar este concepto en el área de la educación? o incluso, ¿cómo promover la inmersión de los docentes en las escuelas de educación básica, para que la teoría y la práctica puedan unirse?

Las respuestas a estas preguntas no consideran la necesidad de definir el concepto de iniciación a la docencia. Durante la implementación, aparentemente, lo más importante es indicar si ser residente se configura para actuar como profesor en Educación Básica o para comenzar las pasantías cuando se inscribe en un curso de licenciatura. En este movimiento, primeramente, el PRP se diseñó como un programa que permitiría la inmersión a tiempo completo en las escuelas de Educación Básica, tanto para los docentes que ya estaban capacitados como para aquellos que estaban interesados en la carrera docente, pero después se definió como un sinónimo de pasantía obligatoria.

Sin embargo, como la historia de la implementación de estos dos programas nacionales de capacitación del profesorado está lejos de concluirse, parece que hay una nueva tendencia, un nuevo movimiento: que el PRP no necesariamente debe vincularse a la pasantía obligatoria, pero, más bien, al período en el que el futuro profesor estudiará para obtener un título, ya que, para ser parte del programa, el estudiante necesariamente debe haber completado al menos el $50 \%$ del curso. En otras palabras, parece que la tendencia del PRP debe configurarse como un complemento de Pibid y, por qué no decirlo, a las prácticas obligatorias.

\section{Referencias bibliográficas}

André, M. (2018). Revista Brasileira de Educação v. 23. https://www.redalyc.org/articulo.oa?id=27554785073. Acceso el: 13/04/2020.

Barbosa, D. y Dutra, N. (2019). Residência pedagógica na formação de professores: uma história de avanços e resistências. Disponible en: http://www.icepsc.com.br/ojs/index.php/gepesvida/article/view/361. Acceso el: 10/04/2020.

Brasil (2007). Edital MEC/CAPES/FNDE. Disponible en: https://capes.gov.br/images/stories/download/editais/Edital_PIBID.pdf. Acceso el: $14 / 09 / 2020$

Brasil (2009). Decreto núero 21, publicado em 30/01/2009. Disponible en: http://capes.gov.br/images/stories/noticia/DOU 30.01.2009 pag 1.pdf. Acceso el: $21 / 06 / 2020$

Brasil (2013). Lei $\mathrm{n}^{\mathrm{o}}$ 12.796, de 4 de abril de 2013. Disponible en: https://www.in.gov.br/materia//asset publisher/Kujrw0TZC2Mb/content/id/30037356/do1-2013-04-05-lei-n-12-796-de4-de-abril-de-2013-30037348. Acceso el: 14/09/2020 
Brasil. (2017). Lançamento do Programa de Residência Pedagógica. Disponible en: http://portal.mec.gov.br/ultimas-noticias/211-218175739/55921-mec-lanca-politicanacional-de-formacao-de-professores-com-80-mil-vagas-para-residencia-pedagogica-em2018. Acceso el: 05/04/2020.

Brasil (2018). Programa de Residência Pedagógica. Disponible en: http://www.capes.gov.br/educacao-basica/programa-residencia-pedagogica. Acceso el: 14/09/2020.

Brasil (2020a). Editais publicados sobre o Programa de Residência Pedagógica. Disponible en: http://www.capes.gov.br/educacao-basica/programa-residencia-pedagogica. Acceso el: 05/04/2020.

Brasil. (2020b). Editais publicados sobre o Programa Institucional de Bolsa de Iniciação à Docência. Disponible en: http://www.capes.gov.br/educacao-basica/capespibid. Acceso el: 05/04/2020.

D’Ambrósio, U. (1999). A História da Matemática: questões historiográficas e políticas e reflexos na Educação Matemática. Disponible en: http://cattai.mat.br/site/files/ensino/uneb/pfreire/docs/HistoriaDaMatematica/Ubiratan D Ambrosio doisTextos.pdf. Acceso el: 14/09/2020.

Faria, J.B. y Diniz-Pereira, J.E. (2019). Residência pedagógica: afinal, o que é isso?. Revista Educação Pública. Cuiabá, v. 28, n. 68, p. 333-356, maio/ago.

Fiorentini, D. y Lorenzato, S. (2007). Investigação em Educação Matemática: percurso teóricos e metodológicos. $3^{\text {a }}$ MECed. Campinas: Autores Associados.

Freitas, H. C. L. (2007). A (nova) política de formação de professores: a prioridade postergada. Educ. Soc., vol. 28, $\mathrm{n}^{\circ}$. 100, out. Especial. Campinas, pp. 1203-1230. Disponible en: http://www.scielo.br/pdf/es/v28n100/a2628100. Acceso el: 03/04/2020.

Gatti et al. (2011). Políticas docentes no Brasil: um estado da arte. Brasília: UNESCO. https://unesdoc.unesco.org/ark:/48223/pf0000212183. Acceso el: 14/09/2020.

Gatti et al. (2014). Um estudo avaliativo do Programa Institucional de Bolsa de Iniciação à Docência (Pibid). Disponible en: http://publicacoes.fcc.org.br/ojs/index.php/textosfcc/issue/view/298. Acesso em 13/04/2020.

Lanner de Moura, A.R. (1995). A medida e a criança pré-escolar. Tese de Doutorado. Faculdade de Educação da UNICAMP. Disponible en: http://repositorio.unicamp.br/simplesearch? query=Anna + Regina + Lanner + de + Moura\&filtername=author. $\quad$ Acceso $\quad$ el: 05/04/2020.

Locatelli, C. (2018). A Política Nacional de Formação Docente: o programa de iniciação à docência no contexto brasileiro atual. Revista Eletrônica de Educação, v. 12, n. 2, p. 308318, maio/ago. 2018. Disponible en: http://www.reveduc.ufscar.br/index.php/reveduc/article/view/2432/706. Acesso em 21/06/2020.

Moreira, M. A. (1990). Pesquisa em Ensino: o vê Epistemológico de Gowin. S.P., E.P.U. 
Nogueira et al. (2011). Trabalho docente e formação de professores: Os professores iniciantes e suas práticas. Debates em Educação, Maceió, v. 3, $\mathrm{n}^{\circ}$ 6, p. 33-52. Disponible en: https://www.seer.ufal.br/index.php/debateseducacao/article/view/622. Acceso el: $14 / 09 / 2020$.

Nogueira ert al. (2013). A docência expressa nas visões e nas vozes de professores iniciantes e acadêmicos: revelações na/da pesquisa-formação. Anais [...] 36 ${ }^{\mathrm{a}}$ Reunião Nacional da ANPEd. Sistema Nacional de Educação e Participação Popular: desafios para as políticas educacionais. Goiânia: ANPEd. Disponible en: https://anped.org.br/biblioteca/item/docencia-expressa-nas-visoes-e-nas-vozes-deprofessores-iniciantes-e-academicos. Acceso el: 14/09/2020.

OCDE. (2006). Professores são importantes: atraindo, desenvolvendo e retendo professores eficazes; relatório de pesquisa. São Paulo: Moderna.

Silva, K.A.C.P. y Cruz, S.P. (2018). A Residência Pedagógica na formação de professores: história, hegemonia e resistências. Momento: diálogos em educação, v. 27, n. 2, p. 227-247, mai./ago. Disponible en: https://periodicos.furg.br/momento/article/view/8062. Acceso el: 08/04/2020.

Silveira, H.E. (2015). Mas, afinal: o que é iniciação à docência?. Atos de Pesquisa em Educação. Blumenau, v. 10, n.2, p.354-368, mai./ago. Disponible en: https://proxy.furb.br/ojs/index.php/atosdepesquisa/article/view/4704. Acceso el: $11 / 04 / 2020$.

Silveira, H.E. (2017). Memórias sobre o Pibid: concepções, criação e dinâmica de funcionamento. Crítica Educativa (Sorocaba/SP), v. 3, n. 2 - Especial, p. 50-62, jan./jun. Disponible

en: http://www.criticaeducativa.ufscar.br/index.php/criticaeducativa/article/view/215. Acesso em 10/04/2020.

UESB (2020). A revista de iniciação à docência. Disponible en: http://periodicos2.uesb.br/index.php/rid/article/view/1597. Acceso el: 11/04/2020. 